\title{
Study Of Clinical Characteristics and Risk Determinants Of Patients With Prosthetic Valve Thrombosis: A Tertiary Care Hospital-Based Observational Study
}

Vaishali Verma, Sanjeev Asotra*, Rajesh Sharma, PC Negi, Arvind Kandoria, Neeraj Ganju, Rajeev Marwaha, Shivani Rao, Kunal Mahajan and Ashu Gupta

Department of Cardiology, I.G.M.C Shimla, India

*Corresponding author: Sanjeev Asotra, Department of Cardiology, I.G.M.C Shimla, India

\begin{abstract}
Introduction: Prosthetic valve thrombosis (PVT) is a rare but serious complication of valve replacement, most often encountered with mechanical prostheses. The present study aims to evaluate patients with prosthetic valve thrombosis in terms of the clinical characteristics, risk determinants, treatment, and outcomes in the prospective observational study.

Material and Methods: The observational study was conducted in a tertiary care center. All patients suspected of having prosthetic valve dysfunction underwent clinical, echocardiography, and fluoroscopic examination. Data regarding risk determinants, clinical characteristics, and response to treatment was categorized on the basis of serial echocardiography and fluoroscopy into 3 classes; complete response, partial response, and failed treatment.

Result: Out of 29 patients 17 (59\%) were females and 12 (41\%) were males. The mean age was 46.6years with an age range from 20-80 years. $15(52 \%)$ patients were post MVR, $9(32 \%)$, post DVR and $5(17 \%)$ were post AVR. Dyspnoea was the presenting complaint in all the patients presenting with valve thrombosis. Mitral valve thrombosis was seen in 19 (65\%), aortic valve thrombosis in $8(28 \%)$, and tricuspid valve thrombosis in $2(7 \%) .86 \%$ of patients had sub-therapeutic INR. Intensification of anticoagulation and thrombolytic therapy was the treatment modalities deployed.76\% of patients received thrombolytic therapy and streptokinase was the most common agent used in $82 \%$ of patients. Majority of patients presented with symptoms of NYHA class III. $62 \%$ of patients showed complete response, $24 \%$ of patients showed partial response, and 4 (14\%) had no response to lytic therapy. 7 out of 29 patients landed up in complications. 4 out of 29 patients had minor bleeds in the form of oral mucosal ooze and haematuria. 3 patients succumbed to complications, one of whom had a major bleed in the form of intracranial hemorrhage and two had worsening of heart failure.
\end{abstract}

Conclusion: The most important risk factor attributed to the occurrence of prosthetic valve thrombosis was non-compliance to treatment leading to subtherapeutic INR. Mitral valve thrombosis was most commonly seen and Fibrinolysis can be considered as effective therapy even in patients presenting with NYHA Class III/IV especially in resource-stricken developing countries.

Keywords: Prosthetic Valve Thrombosis; PVT; Clinical Characteristics; Risk Determinants; Sub-optimal Anticoagulation; Cinefluoroscopy

\section{Introduction}

Prosthetic valve replacement is the mainstay of treatment for patients with severe symptomatic valvular heart disease but in the process, the native valvular disease is exchanged with prosthesisrelated complications. The prosthetic valve dysfunction due to the formation of thrombus, pannus formation, infective endocarditis or paravalvular leak [1]. Prosthetic valve thrombosis (PVT) is a rare but serious complication of valve replacement, most often encountered with mechanical prostheses. PVT has been further divided into Obstructive and Non-Obstructive PVT. The incidence of obstructive PVT for mechanical valves varies between $0.3-1.3-3 \%$ patient years[2,3]. The incidence ranges from $0.1 \%$ to almost $6 \%$ per patient-year for left-sided valves and up to $20 \%$ for tricuspid valves[4]. 
The etiology of PVT is complex and multifactorial. The Suboptimum anticoagulation is the major cause of prosthetic valve thrombosis in addition to type and position of the prosthetic valve, presence or absence of pro-thrombotic states like atrial fibrillation, and ventricular dysfunction[5,6].PVT may present with progressive dyspnea, signs of heart failure, or systemic embolization, or may be incidentally diagnosed during the routine echocardiographic followup. Various modalities aid in the diagnosis of PVT. Trans-thoracic echo (TTE) is the initial screening tool. Trans-esophageal echo (TEE) and Cine-fluoroscopic leaflet mobility profile are further done to establish the pathology[4][6-8]. Various Treatment modalities for treat prosthetic valve dysfunction include intensification of anticoagulation, thrombolytic therapy, surgical thrombectomy, and prosthetic valve replacement. However one should try to prevent this dreadful complication by meticulous monitoring of patient profile and symptomatology at every clinical visit. We conducted a prospective observational study of all patients presenting with PVT. Patients with prosthetic valve thrombosis were diagnosed based on standard criteria and clinical characteristics, risk determinants, treatments, and outcomes were assessed.

\section{Material and Methods}

This was a prospective observational study conducted in a tertiary care institute, The study was duly approved by the institutional review board and ethical committee.

\section{Inclusion Criteria}

All consecutive eligible participants consenting to participate suspected of having prosthetic valve dysfunction based on clinical, echocardiographic, and fluoroscopic findings would be screened for enrollment in the study.

\section{Exclusion Criteria}

Patient not consenting to participate in the study.

\section{Definition Of Pvt}

It was defined on 3 criteria; Clinical, Echocardiographic and Fluoroscopic.

\section{A. Clinical Criteria:}

1) Shortness of breath

2) Features of systemic embolization.

\section{B. Echo Criteria}

1) Increased gradients when compared with the reference values.

2) Prosthetic valve thrombus was recognized as soft and homogeneous, mobile or fixed echo densities located at the valve occluder and/or valve struts. The largest diameter of the thrombus as well as the length of the mobile portion, if present was measured.

3) A diagnosis of pannus formation was made when fixed, bright echo dense structures, sometimes containing focal calcific deposits, were present primarily along the valve ring with extension into the valve ori

\section{Fluoroscopic Criteria}

Each prosthesis was viewed in multiple projections including caudocranial angulations, which allowed proper visualization of prosthesis leaflet motion; that opening and closing angles were calculated. A short cine fluoroscopic film( usually 3 to 10 cardiac cycles) at 50 frames/s was recorded.

\section{Response to Treatment}

Complete Response - Complete success was defined as normal or near-normal transvalvular gradient and restoration of normal leaflet motion on fluoroscopy without any major complications and clinical improvement in symptoms

Partial Response - Partial success was defined as a reduction of $>50 \%$ of transvalvular gradient from the baseline or complete hemodynamic response with major complication or restricted movement of prosthetic valve leaflets on cine fluoroscopy even though the transvalvular gradients completely normalized.

Failed Treatment - Failure of TT was defined as no hemodynamic response even with extended thrombolysis (up to $72 \mathrm{~h}$ ) or death by major complications.

\section{Predefined Complications :}

- $\quad$ All-cause in-hospital mortality.

- $\quad$ Non- fatal minor complications-bleeding without need for transfusion; TIA.

- Non-fatal major complications-ischemic stroke, ICH, embolism(coronary or peripheral), bleeding requiring transfusion.

\section{Various Treatment Regimes for Thrombolytic Agents:}

1. Streptokinase regimen- 2.5 lakh IU intravenous bolus for 30 minutes followed by slow intravenous infusion at the rate of 1 lakh IU/hr

2. Tenecteplase -slow intravenous infusion for $0.5 \mathrm{mg} / \mathrm{kg}$ as an intravenous infusion for up to 72 hours in failed cases.

3. Alteplase- slow intravenous infusion of $0.5 \mathrm{mg} / \mathrm{kg} / \mathrm{hr}$.

\section{Response to Treatment :}

1. Serial echocardiography - Bedside echocardiography was done every six hours and gradients and valve area was recorded

2. Serial fluoroscopy was done every 12 hours and opening and closing angles were determined.

Once the complete response was achieved, infusion of thrombolytic agents was stopped or was continued till 72 hours in cases of failed or partial response. Premature cessation of 
thrombolytic therapy was done in cases of non-fatal minor or major bleeding. After successful thrombolysis, injection of low molecular heparin was given subcutaneously in doses of $1 \mathrm{mg} /$ $\mathrm{kg}$ bid. Acenocoumarol was initiated. Both low molecular weight heparin and acenocoumarol were continued till PT/INR achieved a therapeutic range of 2.5-3.5.

\section{Non-Compliance To Treatment}

1. Regular - patient taking drugs(especially acenocoumarol) daily without fail.

2. Irregular- taking drugs for less than five days a week for the past two days.

3. Stopped- not taken drugs for last one week.

\section{Statistical Analysis}

Data collected were entered into a Microsoft Excel worksheet and analyzed statistically by using Statistical Package for Social Sciences (SPSS Inc., Chicago, IL). Data were presented as mean, standard deviation, and percentages.

\section{Results}

29 patients were diagnosed with prosthetic valve thrombosis during the study period. Table 1 . Shows the clinical characteristics of the patients. The mean age of patients was $46.6 \pm 13.6$ with an age range of $20-80$ years. $59 \%$ of patients were females and the majority of patients $82.7 \%$ belonged to rural areas, literacy rate was $34.48 \%$. 93.1\% of the patients developed thrombosis after more than one year of valve surgery. Thrombosis of the mitral valve was seen in $65 \%$ of patients, $28 \%$ developed thrombosis of the aortic valve and 2 patients had thrombosis of the tricuspid valve. All the valves were bileaflet tilting disc type. Dyspnoea was the presenting complaint in all the patients. $23(79.3 \%)$ patients presented with NYHA class III symptoms,4(13.8\%) presented with NYHA class IV, and 2(6\%) presented with class II symptoms. A suboptimal therapeutic international normalized ratio was found in $86 \%$ of valve thrombosis. NYHA: New York Heart Association, INR: international normalized ratio. Most of the patients had defaulted on their consumption of oral anticoagulant drugs.16(55\%) patients were irregular in drug intake and $6(21 \%)$ had completely stopped taking medications. The most common reason for drug default was non-availability in $13(60 \%)$, inaccessibility in $6(27 \%)$, and ignorance in 3 (13\%)(Table 2).

$22(76 \%)$ patients were treated with thrombolytic therapy,7(24\%) patients received Heparin and intensification of anticoagulation. Streptokinase was used as a thrombolytic agent in 18 (82\%), tenecteplase, and Alteplase in 2 patients each.12(66.6\%) patients who received thrombolysis with streptokinase had a complete response, $4(22.3 \%)$ had a partial response and 2 patients had no response to treatment. Alteplase and Tenecteplase were used in 2 patients. The opening angle and closing angle of the aortic valve were $48.1 \pm 5.4$ and $104.1 \pm 5.5$ pre thrombolysis and $10.7 \pm 0.8$ and $120.8 \pm 3.6$ respectively post successful lysis. The opening angle and closing angle of the mitral valve were $48.7 \pm 6.2$ and $104.3 \pm 4.5$ pre thrombolysis and $10.9 \pm 0.8$ and $119.7 \pm 1.3$ respectively post successful lysis. (Table 3 ) The average mean gradient across stuck aortic valve before thrombolysis was $45.5 \pm 3.2$ and stuck mitral valve was $15.9 \pm 4.1$. Post successful fibrinolysis, the mean gradient across the aortic valve was $20.5 \pm 1.0$ and mitral valve $4.0 \pm 1.1$ (Table 4).

Table 1: Clinical characteristic of the study patients of PVT

\begin{tabular}{|c|c|}
\hline Clinical Characterstic & Numbers(\%) \\
\hline Mean Age (Years) & $46.62 \pm 13.62 \quad(20-80)$ )year \\
\hline Female & $17(59 \%)$ \\
\hline Male & $12(48 \%)$ \\
\hline Literate & $10(34.9 \%)$ \\
\hline Illiterate & $19(65.1 \%)$ \\
\hline Time Between Valve Surgery \&PVT $\geq$ I YEAR & $27(93 \%)$ \\
\hline \multicolumn{2}{|c|}{ Valve Involved } \\
\hline Aortic & $8(28 \%)$ \\
\hline Mitral & $19(65 \%)$ \\
\hline Tricuspid & $2(7 \%)$ \\
\hline \multicolumn{2}{|l|}{ Nyha } \\
\hline CLASS I & NIL \\
\hline CLASS II & $0.90 \%$ \\
\hline CLASS III & $79.30 \%$ \\
\hline CLASS IV & $13.80 \%$ \\
\hline Subtherapeutic INR & $86 \%$ \\
\hline
\end{tabular}


Table 2: Drug compliance and reason of defaults in patients with PVT.

\begin{tabular}{|c|c|}
\hline \multicolumn{2}{|c|}{ Regularity OF Drug Compliance } \\
\hline Regular & $0.7 \%$ \\
\hline Irregular & $16(55 \%)$ \\
\hline Stopped & $06(21 \%)$ \\
\hline \multicolumn{2}{|c|}{ Reason for default } \\
\hline Nonavailability & $13(60 \%)$ \\
\hline Non Afforadabilty & Nil \\
\hline Inaccessibility & $06(27 \%)$ \\
\hline Ignorance & $03(13 \%)$ \\
\hline
\end{tabular}

Table 3: Cine fluoroscopic measurements of PR thrombolysis and post thrombolysis.

\begin{tabular}{|c|c|c|c|c|}
\hline Aortic Valve & Pre Thrombolysis & \multicolumn{3}{|c|}{ Post Thrombolysis } \\
\hline & & Complete Lysis & Partial Lysis & 18 \\
\hline OPENING ANGLE & $48.1 \pm 5.4$ & $10.7 \pm 0.8$ & 113 & 111 \\
\hline CLOSING ANGLE & $104 \pm 5.5$ & $120 \pm 3.6$ & $18.5 \pm 1-9$ & $53 \pm 5.3$ \\
\hline \multicolumn{7}{|c|}{ Mitral valve } \\
\hline OPENING ANGLE & $48.7 \pm 6.2$ & $10.6 \pm 0.9$ & $112 \pm 1.6$ & $103 \pm 2.6$ \\
\hline
\end{tabular}

Table 4: Mean gradient measurement of the stuck valve in aortic and mitral position pre and post thrombolysis.

\begin{tabular}{|c|c|c|c|c|}
\hline \multirow{2}{*}{ Mean Gradients } & \multirow{2}{*}{ Pre Thrombolysis } & \multicolumn{3}{|c|}{ Post Thrombolysis } \\
\cline { 3 - 5 } & & Complete Lysis & \multicolumn{2}{|c|}{ partial lysis } \\
\hline Aortic Valve & $45.5 \pm 3.2$ & $20.5 \pm 1.0$ & 26 & 49 \\
\hline Mitral Valve & $15.9 \pm 4.1$ & $4.0 \pm 1.1$ & $8.5 \pm 1.0$ & $17.0 \pm 4.4$ \\
\hline
\end{tabular}

18(62\%) patients had complete clinical success,7(24\%) had a partial response to treatment, and $4(14 \%)$ had no response to thrombolytic therapy. Duration of therapy was $24 \mathrm{hrs}$ in 11 patients ,18hrs in 8 paients and $72 \mathrm{hrs}$ in 3 patients. 3 patients succumbed to complications, one had massive ICH, and 2 had progressive heart failure. Minor bleeding was seen in 4 patients.

\section{Discussion}

Valvular heart diseases affect more than 100 million people around the globe and are associated with significant mortality and morbidity[3]. Obstruction to prosthetic heart valve could be due to thrombus, pannus formation, vegetation, or patient prosthesis mismatch, Prosthetic Valve thrombosis (PVT) is a pathological entity characterized by thrombus formation on the prosthetic structures, with subsequent PV dysfunction with or without thromboembolism (TE)[6-8]. There is a high prevalence of rheumatic heart disease in young in India and the majority undergo valve replacement surgery at a young age compared to developed countries $[9,10]$. The mean age of our patients was $46.62 \pm 13.6$.most of the studies from the Indian continent of PVT belonging to younger age group in 3rd and 4th decade and female dominance [11-15]. Mean age was $58 \pm 12$ in a study from Montreal [16]. The majority of patients belonged to rural backgrounds and have poor availability of health resources and medical care. This might contribute to drug default by the patient. The majority of patients in our study group were illiterate which might contribute to ignorance which further lead to failure in regular drug intake and cause them to default the drug regimen. Poor drug compliance also emerged as a significant risk factor in studies done by Durrleman et al reported 10 out of 39 were defaulters and Manjula et al reported 23 out of 26 patients were non-compliant $[16,17]$.

The majority of studies have cited sub-therapeutic INR as a major risk factor for the development of PVT which was mainly due to irregular follow-up and poor treatment compliance. Studies from India show variable rates of inadequate anticoagulation ranging from 41.5 To $90.3 \%$ [18-20]. Egbe et al found inadequate anticoagulation in $48 \%$ of patients with PVT [21].

All patients of PVT presented with dyspnea and a muffled click on examination. The most common presentation is dyspnoea in the majority of studies. Most of the patients presented with NYHA class III and Class IV symptoms In our series, most patients were in NYHA class III at presentation $(79.3 \%)$ whereas in series by Nawale et al. maximum patients fell under NYHA class IV (52.4\%) [14]. In the series by Durrleman et al, they reported the maximum number of patients were in class III or IV at presentation (87\%) [16]. Similarly in the study published by Gupta et al, reported that $46.4 \%$ of patients were in NYHA class III and $24.5 \%$ of patients were in NYHA class IV at the time of presentation[18]. 
The mean time between valve replacement and diagnosis of PVT was 32 months in the current series, ranging between 2 to 60 months. In the series published by Nawale et al, this range was 1 month to 96 months,[14] whereas in the series by Durrleman et al this range was between 0 to 120 months[16]. The Mitral valve was the most common valve involved in all the series followed by the aortic valve and the least commonly involved was the tricuspid valve. Mitral valve PVT is 2-3 times more common than aortic. Nawale et al and Durrleman et al reported thrombosis of both aortic and mitral valves in $9.5 \%$ and $15 \%$ of patients respectively[14][16]. Although surgery remains the treatment of choice especially leftsided PVT according to the 2007 Europen society of cardiology and 2008 ACC/AHA guidelines[22,23]. The largest single-center study by Roudaut et al. also showed a better early success rate with surgery and significantly lower complications compared to fibrinolysis[24-29]. Advantages of fibrinolysis include easy availability, relatively low cost, and its simplicity in administration. In a review by Lim et al. involving eight large studies determining the outcome of fibrinolysis in PVT, the success rate of fibrinolytic therapy varied from $58 \%$ to $90 \%$, and the rate of complication was variable[30-33]. Thromboembolism ranged from $1.7 \%$ to $17.6 \%$ and bleeding ranged from 1.7 to $12 \%$. The data from Indian studies also showed a success rate of $59 \%$ to $100 \%$ with an overall success rate of around $78.1 \%$ and mortality rate ranged from 1.9 to $9.5 \%$ with an overall rate of around $5.4 \%$. The rate of major complications ranged from $8.4 \%$ to $18.7 \%$ and minor complications from $2.3 \%$ to $25 \%[19][22][23][25][26]$. In our study complete clinical success was seen in $62 \%$, partial clinical success in $24 \%$ with in-hospital mortality $10.3(\%)$. One patient died of massive ICH and 2 died of progressive heart failure. No patient had an embolic complication. Streptokinase had been used as a first-line drug in most studies[19] [23-26]. Newer fibrinolytics are also equally efficacious for the treatment of PVT [26][31][32].

Streptokinase was used in the majority of patients $66.7 \%$ and tenecteplase and reteplase was used in 2 patients each. In a study conducted by Kathirvel et al. $76.9 \%$ of patients were thrombolysed with STK and 23.1 percent were thrombolysed with TNK[26]. In the study conducted by Hiracharan et al. 86.9 $\%$ of patients were thrombolysed with STK and 13.1\% were thrombolysed with TNK[18]. In our study, $62.1 \%$ of patients were thrombolysed with STK and $6.9 \%$ were thrombolysed with TNK; $6.9 \%$ were thrombolysed with alteplase and $24.1 \%$ of patients were managed with the intensification of thrombolysis. In the current series whenever STK was given, 2.5 lakh IU intravenous bolus for 30 minutes followed by slow intravenous infusion at the rate of 1 lakh $\mathrm{IU} / \mathrm{hr}$ was administered. Extended thrombolysis up to 72 hours was instilled in case of failed response. Total response with streptokinase was observed, in $81 \%$ of patients in a study conducted by Sharma et al[33]. 66.6\% of our patients showed complete response to thrombolysis. $76.2 \%$ and77.5\% of patients showed complete response to thrombolytic therpy in studies conducted by Nawale et al Kathirvel et al [18][26].

\section{Study Limitations}

This was a single-center, prospective observational study with a relatively small number of patients. The newer thrombolytic agent was used in small numbers of patients

\section{Conflicts Of Interest}

There are no conflicts of interest.

\section{Ethical Approval}

The study was approved by the appropriate institutional ethics committee and has been performed as per the ethical standards as laid down in the Declaration of Helsinki.

\section{References}

1. Vesey JM, Otto CM (2004) Complications of prosthetic heart valves. Current Cardiology Reports 6(2): 106-111.

2. Dangas GD, Weitz JI, Giustino G, Makkar R, Mehran R (2016) Prosthetic Heart Valve Thrombosis. J Am Coll Cardiol 68(24): 2670-2689.

3. Horskotte D, D Burckhardt (1995) Prosthetic valve thrombosis. J Heart Valve Dis 4(2): 141-153.

4. Roudaut R, Serri K, Lafitte S (2007) Thrombosis of prosthetic heart valve: Diagnosis and therapeutic considerations. Heart 93(1): 137-142.

5. Lung B, Vahanian A (2011) Epidemiology of valvular heart disease in adults. Nature reviews Cardiology 8(3): 162-172.

6. Habib G, Cornen A, Mesana T, Monties JR, Djiane P, et al. (1993) Diagnosis of prosthetic heart valve thrombosis. The respective values of transthoracic and transoesophageal Doppler echocardiography. Eur Heart J 14(4): 447-455.

7. Vogel W, Stoll HP, Bay W, Fröhlig G, Schieffer H (1993) Cineradiography for determination of normal and abnormal function in mechanical heart valves. Am J Cardiol 71(2): 225-232.

8. Tanis W, Habets J, van den Brink RBA, Symersky P, Budde RPJ, at al. (2014) Differentiation of thrombus from pannus as the cause of acquired mechanical prosthetic heart valve obstruction by non-invasive imaging: a review of the literature. Eur Heart J - Cardiovasc Imaging 15(2): 119129.

9. Inamdar A, Shende S, Inamdar S (2017) Prosthetic valve obstruction: Redo surgery or fibrinolysis? Med J Dr DY Patil Univ 10(3): 246.

10. Cáceres-Lóriga FM, Pérez-López H, Gracias GS, Morlans-Hernández K M (2006) Prosthetic heart valve thrombosis Pathogenisis, Diagnosis and Managment. Int J Cardiol 110(1): 1-6.

11. Krishnan S (2016) Prosthetic heart valve thrombosis: Diagnosis and newer thrombolytic regimes. J Pract Cardiovasc Sci 2(1): 7-12.

12. Biteker M, Altun I, Basaran O, Dogan V, Yildirim B, et al. (2015) Treatment of Prosthetic Valve Thrombosis: Current Evidence and Future Directions. J Clin Med Res 7(12): 932-936.

13. Akbarian M, Gerald Austen W, Yurchak PM, Scannell JG (1968) Thromboembolic Complications of Prosthetic Cardiac Valves 37(5): 826-831.

14. Negi PC, Sondhi S, Asotra S, Mahajan K, Mehta A (2019) Current status of rheumatic heart disease in India. Indian Heart J 71(6): 86-90.

15. Kumar AS (2020) Surgical options in rheumatic heart disease; an Indian surgeon's perspective. Asian Cardiovasc Thorac Ann 28(7): 371-373.

16. Mansuri Z, Sharma V, Jain S, Prajapati J, Bhatia S, et al. (2020) Clinical profile of prosthetic heart valve thrombosis and outcome analysis 
of fibrinolytic therapy versus surgical management: A single-center experience. Heart India 8(2): 74-79.

17. Patil S, Shetty N, Ramalingam R, Rudrappa MMB, Manjunath CN (2019) Study of prosthetic heart valve thrombosis and outcome after thrombolysis. Int J Res Med Sci 7: 1074-1078.

18. Hirachan A, Roka M, Prajapati D, Adhikari CM, Bishal KC, et al. (2017) Prosthetic valve thrombosis in a territory cardiac centre. Nepal Heart $j$ 14(1): 9-11.

19. Nawale JM, Chaurasia AS, Nalwale DD, Abdagare NK (2018) Clinical profile of patients with prosthetic valve thrombosis treated with fibrinolysis. J Pract Cardiovasc Sii 4(2): 109-115.

20. Khan HS, Ijaz Z, Ali M, Saif M, Ishary U, Kamal A, Ikram U, Sattar RA, Malik J. Clinical outcomes of prosthetic valve thrombosis Cureus 12(6): e8760.

21. Durrlemann N, Pellerin M, Bouchard D, Herbert DY, Cartier R, at al. (2004) Prosthetic Valve thrombosis: Twenty years' experience at Montreal heart institute. J Thorac Cardiovasc Surg 127(5): 1388-1392.

22. Manjula M, Rangan K, Manjunath CN (2017) Clinical profile and outcome of recurrent prosthetic heart valve thrombosis in a tertiary care cardiology unit. Indian Heart J 69.

23. Gupta D, Kothari SS, Bahl VK, Goswami KC, Manchanda SC, et al. (2000) Thrombolytic therapy for prosthetic valve thrombosis: Short- and longterm results. Am Heart J 140(6): 906-916.

24. Karthikeyan G, Math RS, Mathew N, Shankar B, Kalaivani M, et al. (2009) Accelerated infusion of streptokinase for the treatment of left-sided prosthetic valve thrombosis: A randomized controlled trial. Circulation 120(12): 1108-1014.

25. Vasan RS, Kaul, Sanghavi S, Kamlakar T, Negi PC, et al. (1992) Thrombolytic therapy for prosthetic valve thrombosis: A study based on serial Doppler echocardiographic evaluation. Am Heart j 123(6): 15751580.
26. Kathirvel D, Justin Paul G, Govidrajam PK, Palanisamy G, Gnanavelu G, et al. (2018) Tenecteplase versus streptokinase thrombolytic therapy in patients with mitral prosthetic valve thrombosis. Indian Heart J 70(4): 506-510.

27. Egbe AC, Pislaru S V, Pellikka PA, Poterucha JT, Schaff H V, et al. (2015) Bioprosthetic Valve Thrombosis Versus Structural Failure: Clinical and Echocardiographic Predictors. J Am Coll Cardiol 66(21): 2285-2294.

28. Vigoda MM, Sweitzer B, Miljkovic N, Arheart KL, Messinger S, Candotti K et al. (2011) 2007 American college of cardiology/American Heart Association(ACC/AHA) guidelines on perioperative cardiac evaluation are usually incorrectly applied by anesthesiology residents evaluating simulated patients. Anaesth Analg 112(4): 940-949.

29. Roudaut R, Lafitte S, Roudaut MF, Reant P, Pillois X, et al. (2009) Management of prosthetic heart valve obstruction: Fibrinolysis versus surgery. Early results and long-term follow-up in a single-center study of 263 cases. Arch Cardiovasc Dis 102(4): 269-277.

30. Lim WY, Lioyd G, Bhattacharyya S (2017) Mechanical and surgical bioprosthesis valve thrombosis. Heart 103(24): 1934-1941.

31. Sharma V, Singh R, Mishra R, Arora AP, Gupta LC, YadavaOP, et al. (2012) Use of Tenecteplllase for left-sided prosthetic valve thrombosis: A randomized controlled trial. J Assoc Physicians India 60: 55-58.

32. Kumar BM, Paul GJ, Swaminathan N, Venkatesan S, Hussain GZ, et al. (2017) Assessment of hemodynamic and clinical response in thrombolytic therapy for prosthetic valve thrombosis. Indian Heart J 69(S2): S6.

33. Sharma KH, Mewada NM (2010) Efficacy and safety of streptokinase in prosthetic valve thrombosis(total 5 year clinical registry). Ind J Pharmacol 42(5): 330-331.

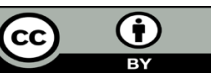

This work is licensed under Creative Commons Attribution 4.0 License

To Submit Your Article Click Here:

Submit Article

DOI: 10.32474/ACR.2021.03.000170

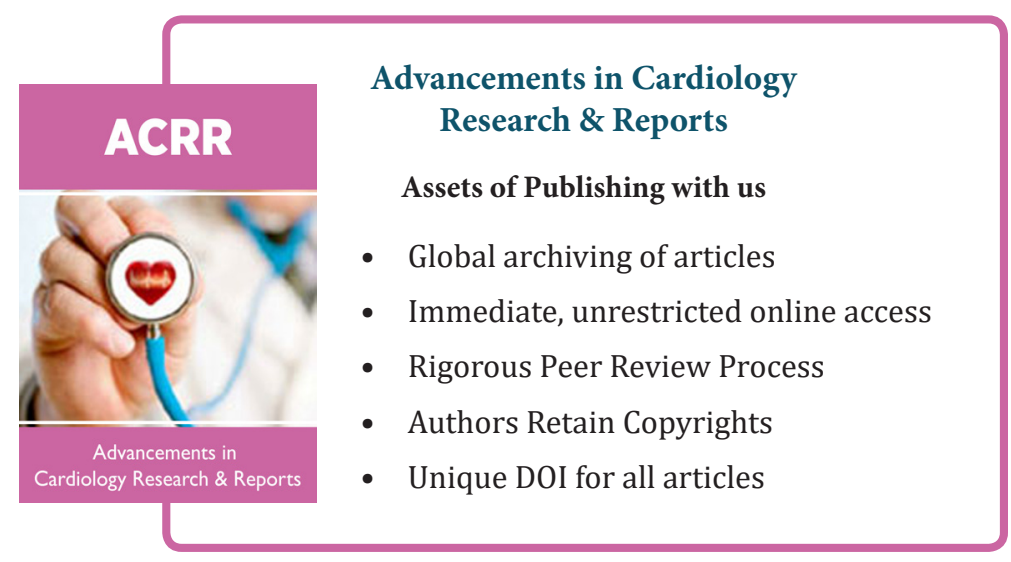

